\title{
Splicing up repair mechanisms
}

The extent to which axons regenerate after injury is typically much greater in the peripheral nervous system (PNS) than in the CNS; however, the factors that determine regenerative capacity are poorly understood. Song et al. now reveal that RNA 3'-terminal phosphate cyclase (Rtca), an enzyme that regulates RNA processing and repair, inhibits regeneration of sensory neuron axons within the CNS in Drosophila melanogaster.

In wild-type flies, the axons of class IV dendritic arborization (da) neurons can regenerate if they are severed in the periphery, whereas limited regrowth is observed if the axons are severed in the CNS. By damaging these axons in fly larvae, the authors aimed to identify genes that are important for axon regeneration. They found that, in fly larvae carrying a loss-of-function mutation in Rtca, the axons exhibited a much more robust regenerative response to damage in the CNS. Conversely, overexpression of Rtca in class IV da neurons reduced axon regeneration after injury in the PNS, confirming that Rtca inhibits axon regeneration.

Rtca regulates RNA splicing and repair by converting the 3 '-phosphate end of a spliced or damaged RNA into a $2^{\prime}, 3^{\prime}$-cyclic phosphate, which possibly prevents its ligation into the mature processed RNA. To further investigate the involvement of RNA processing pathways in axon regeneration, the authors examined axon regrowth after injury in fly larvae deficient in the gene encoding Archease, a protein that catalyses
RNA ligation. Loss of function of Archease impaired the regeneration of the class IV da neuron axons in the periphery. Furthermore, loss of function of Archease abolished the positive effect of the Rtca mutation on axon regeneration in the CNS.

Which RNAs are targeted by the Rtca-regulated RNA splicing and/or repair pathway? As part of the intracellular response to cellular stress, X-box-binding protein 1 (Xbp1) mRNA undergoes unconventional splicing to generate a transcriptional activator - Xbp1s - that promotes homeostasis. The authors showed that induction of a stress response in fly larvae via a heat shock increased the levels of Xbp1s: this induction was enhanced in Rtca mutants but was reduced in Archease mutants, confirming that these enzymes regulate $X b p 1$ splicing.

The authors found that flies deficient in $X b p 1$ exhibited reduced peripheral regeneration of class IV da neuron axons, whereas overexpression of $\mathrm{X} b p 1 s$ increased axon regeneration in the CNS. $X b p 1$ loss of function also reduced the regeneration-enhancing effects of Rtca loss in axons in the CNS.

These findings indicate that the RNA repair and splicing pathway regulated by Rtca and Archease has an important role in axon regeneration and provide insight into the downstream mechanisms. There is early evidence that the role of this pathway is conserved - the authors showed that the regeneration of retinal ganglion cell axons after optic nerve crush is enhanced in mice deficient in Rtca - suggesting that this pathway may provide new potential targets for therapeutic intervention in nerve injury.

Katherine Whalley

ORIGINAL RESEARCH PAPER Song, Y. et al. Regulation of axon regeneration by the RNA repair and splicing pathway. Nat. Neurosci. 18, 817-825 (2015)

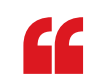

in fly larvae carrying a lossof-function mutation in Rtca, the axons exhibited a much more robust regenerative response to damage in the CNS

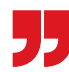

